\title{
LE CONCEPT D'ATOME DEPUIS CENT ANS
}

\author{
A. KASTLER \\ Laboratoire de Physique, Ecole Normale Supérieure \\ 24, rue Lhomond, Paris, France
}

\begin{abstract}
Résumé. - Les chimistes ont adopté dès le milieu du $\mathrm{xIX}^{\mathbf{e}}$ siècle la théorie atomique alors que la plupart des physiciens, sous l'influence de la philosophie positiviste, lui étaient restés hostiles. Cependant, la théorie cinétique des gaz a été édifiée dès la $2^{\mathrm{e}}$ moitié du XIXe siècle par Clausius et par Maxwell et a conduit Boltzmann à développer la mécanique statistique et à donner une interprétation probabiliste de l'entropie qui a conduit Planck à la découverte du quantum d'action. Le nombre d'Avogadro dont l'ordre de grandeur avait été indiqué dès 1865 par Loschmidt a pu être déterminé entre 1870 et 1920 par une dizaine de méthodes indépendantes avec une précision croissante. En France, Jean Perrin a exercé, par ses recherches personnelles et par ses écrits, un rôle déterminant pour faire triompher la théorie atomique. Paul Langevin a montré que les méthodes de Boltzmann permettaient de donner une interprétation théorique des lois du paramagnétisme établies expérimentalement par Pierre Curie. La technique des jets atomiques, inventée en 1911 à Paris par Louis Dunoyer, a conduit à des développements qui ont joué un rôle fondamental en Physique atomique : expérience de Stern et Gerlach, spectroscopie des radiofréquences par la méthode de Rabi, découverte du Lamb-shift. L'avènement de la mécanique ondulatoire et les développements de la mécanique quantique ont montré que les atomes, objets de la réalité microphysique, ont des propriétés très différentes des objets macroscopiques, propriétés qui tiennent au principe d'indétermination. Ce principe oblige le physicien à renoncer aux concepts de discernabilité des particules et de leur permanence dans l'espace-temps, mais en revanche la mécanique ondulatoire introduit en physique atomique les notions de phase et de cohérence qui se traduisent par des propriétés nouvelles de la matière à l'échelle macroscopique.
\end{abstract}

\begin{abstract}
As far back as the middle of the 19th century, chemists adopted the atomic theory but it still met with the hostility of most of the physicists influenced by the positivist philosophy. However, the kinetic theory of gases had been set up in the second half of the 19th century by Clausius and Maxwell and led Boltzmann to the development of statistical mechanics and to a probabilistic interpretation of entropy ; this, in turn, led Planck to the discovery of the quantum of action. The Avogadro number, the magnitude of which had already been indicated by Loschmidt in 1865 , was determined betwen 1870 and 1920 by about ten independent methods of increasing accuracy. In France, Jean Perrin, through his personal researches and his writings played an important role in the success of atomic theory. Paul Langevin pointed out that the methods of Boltzmann allowed a theoretical interpretation of the laws of paramagnetism, experimentally established by Pierre Curie. The technique of atomic jets, invented, in Paris; in 1911, by Louis Dunoyer, led to developments which played a fundamental role in atomic physics, e. g. the Stern and Gerlach experiment, the spectroscopy of radiofrequencies by the Rabi method and the discovery of the Lamb-shift. The advent of the wave mechanics of Louis de Broglie and the developments of quantum mechanics showed that atoms, microphysically real objects, due to the principle of indetermination. This principle compels physicists to give up the concepts of the distinguishability of particles and of their permanence in space-time. But on the other hand the wave mechanical ideas of phase and coherence, introduced in atomic physics, lead to new properties of matter on the macroscopic scale.
\end{abstract}

1. L'atomisme naissant. - Il y a cent ans, la philosophie positiviste, inaugurée par Auguste Comte, dominait la pensée scientifique. Cette tendance était représentée, en France, par Pierre Duhem, en Allemagne par Wilhelm Ostwald, en Autriche par Ernst Mach. Ils défendaient l'exigence que la Science devait se limiter à une "phénoménologie ", c'est-à-dire à une description des relations entre phénomènes directement observables par nos sens, et que tous les «modèles » hypothétiques utilisés pour expliquer les phénomènes observables devaient être éliminés de la pensée scientifique. Parmi ces hypothèses à éliminer se trouvait celle de la structure atomique de la matière. Comme exemple de science positiviste fut prônée "l'énergétique» qui opérait avec les concepts de «chaleur », «d'énergie », «d'entropie » définis à partir des données macroscopiquement observables et qui renonçait à tout modèle mécanique de la chaleur. Ce point de vue était d'ailleurs légitime et devait se révéler fécond. C'est l'analyse critique faite par Mach des notions d'espace et de temps qui devait conduire Einstein aux révisions profondes qui ont donné naissance à la théorie de la relativité. Mais ce point de vue devait avoir également un côté stérile qui se manifestait dans la véhémence dogmatique avec laquelle Mach et Ostwald s'opposaient à l'atomisme naissant qui avait trouvé droit de cilé en chimie grâce à l'œuvre de Dalton. 
Les chimistes avaient reconnu la commodité et l'intérêt de la notation atomique dès le milieu du xix ${ }^{\mathrm{e}}$ siècle. Mais, chez eux aussi, cette évolution ne s'était pas faite sans heurt. En France, Charles Gerhardt, originaire de Strasbourg et Auguste Laurent, originaire de Langres, défenseurs de la théorie atomique, s'étaient heurtés à l'hostilité de Jean-Baptiste Dumas, mais vers la fin de leur vie - ils sont morts jeunes entre 1850 et 1860 - la théorie atomique avait triomphé en chimie. En 1865, Kékulé devait établir sa célèbre formule de l'anneau benzénique et en 1874 Van't Hoff devait écrire son illustre mémoire sur la relation entre le pouvoir rotatoire et la structure des molécules dans l'espace. La stéréochimie était née et Louis Pasteur devait s'en inspirer dans ses travaux.

Les cristallographes de leur côté avaient beaucoup contribué à consolider le concept de structure : RenéJust Hauÿ, mort en 1822, et Auguste Bravais, mort en 1863, avaient interprété les propriétés des cristaux par l'idée d'un empilement répétitif régulier d'éléments constitutifs, les mailles cristallines, formant un réseau périodique à trois dimensions, idées qui devaient être reprises si heureusement, en 1912, par Max Von Laue.

Cependant les physiciens, entre les années 1870 et 1900 , étaient restés la plupart, sous l'influence du positivisme, hostiles ou indifférents à l'égard de la théorie atomique. Max Planck lui-même avoue dans ses souvenirs qu'il avait conservé, jusqu'en 1900, une attitude non seulement d'indifférence, mais de rejet à l'égard de la théorie atomique et que Boltzmann lui en avait voulu. Ce dernier devait d'ailleurs surtout se plaindre amèrement de l'hostilité ouverte et souvent sarcastique d'Ostwaid et de Mach à l'égard de ses travaux.

Cependant, dès 1738, le physicien bâlois Daniel Bernoulli avait attribué la pression qu'un gaz exerce sur une paroi à l'effet des chocs des molécules contre cette paroi, et la découverte du mouvement brownien, en 1827, devait donner un appui considérable à cette hypothèse. Dans les papiers posthumes que nous a laissés Sadi Carnot, emporté en 1832 par l'épidémie de choléra, se trouve le passage suivant : " La chaleur n'est autre chose que la puissance motrice ou plutôt le mouvement qui a changé de forme, c'est un mouve.. ment. " Carnot avait donc, sans aucun doute, l'intuition de l'agitation thermique animant les particules constitutives des objets matériels. C'est pendant la période qui s'étend de 1858 à 1890 que trois hommes : Clausius, Maxwell et Boltzmann, devaient établir sur des bases théoriques sûres ce que nous appelons aujourd'hui la «théorie cinétique des gaz » et «la mécanique statistique $"$.

En 1858, Rudolphe Clausius, interprétant l'énergie interne d'un gaz par l'énergie cinétique des molécules qui s'entrechoquent, introduisit la notion de «libre parcours moyen $\gg l$ et calcula la valeur de cette grandeur en fonction du nombre $n$ de molécules par unité de volume et du «diamètre de choc » $D$ des molécules, considérées comme des sphères dures. Il trouva

$$
l=\frac{1}{n \times \pi D^{2}} .
$$

En 1860 Maxwell montra qu'il faut corriger cette formule et écrire

$$
l=\frac{1}{\sqrt{2}} \cdot \frac{1}{n \pi D^{2}}
$$

et dans un mémoire devenu célèbre il établit la loi de répartition des vitesses moléculaires qui porte son nom :

$$
\frac{1}{n} \frac{\mathrm{d} n_{v}}{\mathrm{~d} v}=4 \pi\left(\frac{M}{2 \pi R T}\right)^{3 / 2} \cdot v^{2} \exp \left(-\frac{\frac{1}{2} M v^{2}}{R T}\right) .
$$

Rappelons que le calcul de la pression - attribué à l'impact des molécules sur la paroi - lui donna l'expression :

$$
p=n \cdot \frac{m v^{2}}{3}
$$

en fonction du carré moyen $\overline{v^{2}}$ des vitesses de translation moléculaires et de la masse $m$ d'une molécule individuelle. En introduisant le volume molaire $V$ et la masse molaire $M=V . n m$, la relation précédente prend la forme

$$
\frac{1}{2} \overrightarrow{M v^{2}}=\frac{3}{2} P V=\frac{3}{2} R T
$$

qui permet le calcul des valeurs numériques des vitesses quadratiques moyennes $v^{*}=\sqrt{v^{2}}$.

On obtient ainsi dans les conditions normales $v^{*}=460 \mathrm{~ms}^{-1}$ pour $\mathrm{O}_{2}$ et $v^{*}=1840 \mathrm{~ms}^{-1}$ pour $\mathrm{H}_{2}$, valeurs qui ont l'ordre de grandeur des vitesses du son dans ces gaz. Ces résultats permettaient de comprendre les lois d'effusion des gaz par un orifice, établies expérimentalement par Graham.

Nous devons remarquer que pour calculer $v^{*}$, il n'est pas nécessaire de connaître le «nombre d'Avogadro » $N=n V$, nombre de molécules par moléculegramme, qui doit être le même pour tous les gaz d'après la loi d'Avogadro.

Les efforts essentiels des physiciens atomistes, dans le deuxième tiers du $\mathrm{XIX}^{\mathrm{e}}$ siècle et au début de notre siècle, étaient tendus vers la connaissance de ce nombre.

Maxwell devait montrer que les chocs entre molécules déterminaient la viscosité du gaz et que le coefficient de viscosité $\eta$ pouvait s'exprimer en fonction du diamètre de choc $D$ par la relation :

$$
\eta=\frac{m \cdot \bar{v}}{4 \pi D^{2}}=\bar{v} \cdot \frac{M}{4 \pi D^{2} \cdot N}
$$

dans laquelle $\bar{v}$ représente la vitesse moyenne reliée à la vitesse quadratique moyenne $v^{*}$ par

$$
\frac{\bar{v}}{v^{*}}=\sqrt{\frac{8}{3 \pi}} .
$$

Il est remarquable que cette expression de $\eta-$ qui 
dépend de la température $T$ par l'intermédiaire de la vitesse moyenne $\bar{v}$ - ne dépende pas de la densité $n$ du gaz. C'était là un résultat surprenant, et Maxwell a tenu, par des mesures expérimentales, à vérifier cette indépendance. Malgré cet indéniable succès de la théorie atomique, celle-ci ne restait qu'une belle hypothèse tant qu'on ignorait le nombre et les dimensions réels des molécules.

La possibilité de déterminer ce nombre constituait le critère qui devait finalement décider du sort de la théorie. Le premier pas dans cette voie fut fait, en 1865, par le physicien autrichien Loschmidt. Les mesures de Maxwell du coefficient de viscosité $\eta$ d'un gaz faisaient connaître le produit $D^{2} N$ des deux inconnues $D$ et $N$. Une deuxième relation était nécessaire pour pouvoir déterminer ces inconnues. Lorsque, dans un empilement compact, les molécules supposées sphériques sont jointives, le volume occupé par une molécule-gramme doit être de l'ordre de

$$
V=N \cdot D^{3} \text {. }
$$

Identifiant cette expression avec le volume molaire à l'état liquide, Loschmidt montra que $D$ devait être de l'ordre de $10^{-8} \mathrm{~cm}$ (un angström) et $N$ de l'ordre de $10^{23}$. C'est la raison pour laquelle, dans les pays de langue germanique, on a l'habitude de désigner par le terme de « nombre de Loschmidt» le nombre $N$ que nous appelons «nombre d'Avogadro".

Cette première détermination fut améliorée lorsqu'en 1873 , il y a donc juste cent ans, Van der Waals proposa d'adopter l'équation d'état des gaz

$$
\left(p+\frac{a}{V^{2}}\right)(V-b)=R T
$$

et montra que pour une mole le «covolume » $b$ devait être égal à

$$
b=\frac{2}{3} \pi D^{3} N \text {. }
$$

Les déterminations expérimentales du coefficient de viscosité d'une part, du coefficient $b$ de l'équation d'état d'autre part, devaient ainsi permettre, en combinant les résultats, d'obtenir $N$ et $D$ pour divers gaz. L'application aux trois gaz $\mathrm{O}_{2}, \mathrm{~N}_{2}$ et $\mathrm{CO}$ permettait alors d'assigner à $N$ des valeurs comprises entre 0,4 et $0,5 \times 10^{24}$.

Ce fut finalement le fait qu'une dizaine de méthodes différentes, basées sur des phénomènes physiques divers, fournissait, au cours du demi-siècle 1870-1920, des valeurs numériques convergentes pour $N$, qui imposa dans l'esprit de tous les physiciens, la conviction de la réalité atomique. L'anecdote veut qu'Ernst Mach se soit converti le jour - en 1910 - où on lui montra au spinthariscope les scintillations produites sur un écran fluorescent par les rayons $\alpha$ du radium.

2. L'œuvre de Boltzmann. - Nous reviendrons plus loin sur la détermination du nombre d'Avogadro. Mais je voudrais auparavant insister sur le rôle qu'a joué, pour le développement de la théorie atomique, l'auvre scientifique de Ludwig Boltzmann dont les publications se sont échelonnées entre 1868 et 1897 et qui, dès 1877 , a proposé une interprétation probabiliste du concept d'entropie. Boltzmann est le fondateur de la mécanique statistique. On lui doit l'introduction, en physique, de la notion de probabilité d'une distribution de molécules et de la définition de «complexions» également probables. Pour ce faire, il considérait l'espace des vitesses de Maxwell dans lequel chaque molécule est représentée par un point, extrémité de son vecteur vitesse. Pour pouvoir définir une « complexion » Boltzmann divisait l'espace des vitesses en "cellules " $\left({ }^{1}\right)$, et considérait le nombre $n_{i}$ de molécules dans chaque cellule et leur numéro d'ordre. Il admettait que tout échange de deux molécules entre des cellules différentes (et tout déplacement d'une molécule d'une cellule dans une cellule voisine de même énergie) donnait naissance à une complexion nouvelle, mais que tout échange de molécules à l'intérieur d'une même cellule (et tout déplacement d'une molécule à l'intérieur d'une cellule) laissait la complexion inchangée. Ces définitions étant posées, il devenait possible d'appliquer l'analyse combinatoire au calcul du nombre de complexions correspondant à un "état» donné du gaz, état défini par la répartition des molécules en fonction de l'énergie. La probabilité $W$ d'un tel état était représentée par le nombre de complexions correspondantes.

La subdivision de l'espace des vitesses en "cellules», éléments d'extension finis de cet espace, était sans doute dans l'esprit de Boltzmann un artifice de calcul, et on a coutume de dire qu'on peut — en mécanique statistique classique - faire tendre vers zéro l'extension de ces cellules. Mais il est facile de voir qu'une telle opération rendrait infinie la valeur de la probabilité de chaque état et que la notion même de probabilité perdrait dans ce cas sa signification. En fait, en inventant ces "cellules» Boltzmann a fait le premier pas vers la théorie des quanta, et Max Planck s'en est directement inspiré dans sa théorie du rayonnement du corps noir. En schématisant nous pouvons dire que les deux hommes se trouvaient confrontés à deux problèmes similaires : répartir un «contenu » dans un «contenant» et définir une probabilité de la répartition.

Pour Boltzmann le contenu était le gaz et possédait une structure discontinue. Il était formé d'un nombre déterminé de molécules, mais le «contenant», l'espace des vitesses, avait une structure continue. Pour pouvoir appliquer l'analyse combinatoire à son problème, Boltzmann s'était vu obligé de quantifier le contenant. Pour Planck, en 1900, il s'agissait de répartir l'énergie de rayonnement dans une cavité. Ici c'était le contenant, la cavité, qui possédait déjà une structure discontinue ; elle se présentait sous forme de modes d'ondes stationnaires et Lord Rayleigh venait d'apprendre

(1) L. Boltzmann, Gastheorie 1896, p. 40, Boltzmann n'avait pas encore l'habitude de considérer l'espace des phases qui a été introduit par Gibbs. 
comment en établir le décompte, alors que c'était le contenu, le rayonnement qui était supposé être de structure continue. Pour faire comme Boltzmann, Planck s'est donc vu obligé de quantifier le contenu en introduisant les «éléments d'énergie $\&$ ». On voit que les deux problèmes étaient analogues ou plutôt symétriques. Il est remarquable qu'à partir des données expérimentales disponibles sur le rayonnement du corps noir, Planck ait pu calculer la valeur non seulement de la constante d'action $h$, mais de la constante des gaz $k=R / N$ d'où il déduisait pour le nombre d'Avogadro $N$ une valeur très peu différente de la valeur actuellement admise. Cette valeur était

$$
N=6,17 \times 10^{23} .
$$

L'œuvre de Boltzmann avait abouti au postulat de la proportionnalité entre le logarithme de la probabilité $W$, grandeur de mécanique statistique et l'entropie $S$, grandeur thermodynamique, relation mise par Planck sous la forme $S=k \ln W$. Cette œuvre devait être complétée au début de ce siècle par l'analyse théorique des fluctuations faite par Einstein et par Smoluchowski.

3. Jean Perrin. - Il convient maintenant, dans ce survol rétrospectif, de parler de la contribution des physiciens français au développement de la physique atomique. Je dois évoquer deux grandes figures: Paul Langevin et Jean Perrin dont nous venons de fêter les centenaires de naissance et dont les travaux se sont inspirés directement de l'œuvre de Boltzmann. En prenant comme paramètre variable d'une distribution moléculaire l'énergie $E$, Boltzmann avait mis la loi de distribution sous la forme aujourd'hui classique :

$$
\frac{n_{1}}{n_{2}}=\exp \left(-\frac{E_{1}-E_{2}}{k T}\right)
$$

$E_{1}$ et $E_{2}$ étant deux niveaux d'énergie moléculaire et $n_{1}$ et $n_{2}$ les nombres d'occupation des «cellules » à ces niveaux. Un exemple d'application classique de cette relation est l'étude de l'équilibre géothermique d'un gaz dans une atmosphère isotherme soumise au champ de pesanteur, le poids d'une molécule étant $f=m g$, et la différence d'énergie potentielle étant $\left(E_{1}-E_{2}\right)=m g\left(Z_{1}-Z_{2}\right)=m g Z$ en désignant par $Z$ la différence d'altitude entre ces niveaux.

En introduisant les densités $\rho_{1}$ et $\rho_{2}$ aux deux niveaux, la relation prend la forme :

$$
\frac{\rho_{1}}{\rho_{2}}=\exp \left(-\frac{m g Z}{k T}\right)=\exp \left(-\frac{M g}{R T} Z\right),
$$

et l'on peut définir une hauteur d'échelle $Z_{0}$, telle que

$$
\frac{\rho_{1}}{\rho_{2}}=e^{-1} \quad \text { et } \quad Z_{0}=\frac{R T}{M g}=\frac{k T}{m g} .
$$

Pour l'atmosphère terrestre $(M \approx 29 \mathrm{~g})$ on trouve dans les conditions normales $Z_{0}=8,8 \mathrm{~km}$.

Jean Perrin devait directement s'inspirer de cette relation pour étudier la répartition en altitude des grains d'une suspension colloïdale dans un liquide.

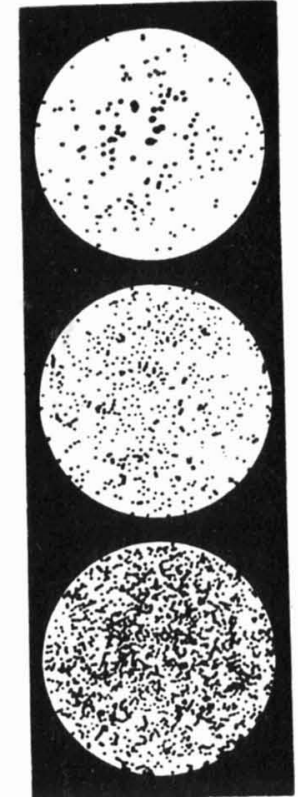

Fig. 1. - Nombre de grains d'une suspension colloïdale à différentes altitudes (d'après Jean Perrin).

Une étude au microscope permet de décompter les nombres de grains aux différentes altitudes et de mettre en évidence la raréfaction progressive du nombre de grains lorsqu'on s'élève en altitude. Perrin montra que la loi de raréfaction est de forme exponentielle et que la formule ci-dessus lui est directement applicable. Il tenait compte de la poussée du liquide en remplaçant $g$ par

$$
g^{\prime}=g\left(1-\frac{\delta}{\Delta}\right)
$$

$\delta$ étant la masse volumique du liquide et $\Delta$ celle de la suspension. La mesure directe des diamètres des grains sphériques au microscope permet de connaitre leur masse $m$, de sorte que la mesure de $Z_{0}$ aboutit à la connaissance numérique de la constante $k$, d'où l'on déduit $N=R / k$.

De nombreuses déterminations faites par Jean Perrin et ses collaborateurs entre 1908 et 1913 conduisirent à la valeur $N=6,8 \times 10^{23}$.

En travaillant toujours sur des suspensions colloïdales, Jean Perrin a également mis au point d'autres méthodes de détermination de $N$. Il a utilisé, en particulier, dans ce but, les lois du mouvement brownien. Einstein avait montré que le carré moyen $\overline{X^{2}} \mathrm{du}$ déplacement d'une particule était proportionnel au temps $t: \overline{X^{2}}=2 \Delta . t$ et que le coefficient de proportionnalité $\Delta$, appelé "coefficient de diffusion 》 s'exprime en fonction du rayon $a$ des grains et du coefficient de viscosité $\eta$ du liquide par la relation :

$$
\Delta=k T \frac{1}{6 \pi a \eta} \text {. }
$$

La détermination de $\Delta$ permet donc d'atteindre de nouveau la constante $k$ et par suite le nombre $N$. 
L'application de cette méthode donna à Jean Perrin la valeur $N=6,4 \times 10^{23}$.

Dans le livre "Les Atomes » qui connut une première édition en 1913, Jean Perrin donna un compte rendu synthétique de ses recherches et confronta les résultats qu'il avait obtenus avec les résultats d'autres méthodes.

Ce livre a été le livre de chevet des jeunes physiciens de ma génération.

Parmi les autres méthodes de mesures de $N$ non encore mentionnées, il convient de citer la mesure de la charge électrique élémentaire par Millikan, la mesure de la charge transportée par les rayons $\alpha$ du radium par Rutherford, et la mesure de l'intensité de la lumière diffusée par les gaz.

L'analyse par Lord Rayleigh, au début de ce siècle, du phénomène de la diffusion moléculaire de la lumière par les fluides, mit en évidence le fait que l'existence d'une telle diffusion, se manifestant dans l'observation ancestrale de la couleur bleue du ciel diurne, constitue une preuve directe de la structure discontinue de la matière. Des mesures de laboratoire de l'intensité de la lumière diffusée par les gaz faites par Jean Cabannes en 1920, donnèrent pour le nombre d'Avogadro la valeur $N=6,5 \times 10^{23}$.

C'est la convergence de tous ces résultats obtenus par des méthodes très différentes et indépendantes les unes des autres qui devait, comme nous l'avons

\begin{tabular}{|c|c|}
\hline PHEXMITES OHER & $\frac{x}{(1)=2}$ \\
\hline 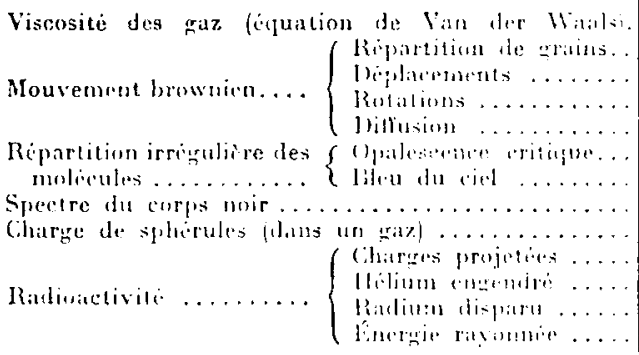 & 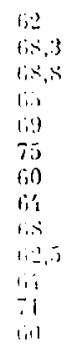 \\
\hline
\end{tabular}

FIG. 2. - Tableaux synoptiques des résultats de mesure du nombre d'Avogadro par différentes méthodes (d'après Jean Perrin).

déjà mentionné, convaincre les physiciens de la réalité des atomes et des molécules. La valeur aujourd'hui admise pour le nombre d'Avogadro est

$$
N=6,02252\left( \pm 28 \times 10^{-5}\right) \times 10^{23} .
$$

4. Paul Langevin. - Paul Langevin devait, de son côté, faire une belle application de la relation de Boltzmann en développant, en 1905, sa théorie du paramagnétisme. Pierre Curie avait montré que la susceptibilité magnétique des corps paramagnétiques était inversement proportionnelle à la température absolue. Langevin attribua ce résultat ì l'existence de moments magnétiques permanents $N$ dont les ions ou atomes paramagnétiques étaient porteurs. Ces moments tendent à s'orienter sous l'action du champ mais cette orientation est contrecarrée par l'effet désorientant de l'agitation thermique. Si nous appelons 0 l'angle entre le vecteur $\boldsymbol{\mu}$ et le vecteur champ $\mathbf{B}$, l'énergie potentielle d'interaction est donnée par l'expression

$$
E=-\mu B \cos \theta .
$$

Il convient d'introduire cette expression dans la relation de Boltzmann. Langevin montra que dans ces conditions le moment molaire mesuré dans un champ $B$ à une température $T$ doit être donné par la relation :

$$
\mathscr{M}=\mathscr{M}_{\mathrm{s}}\left[\operatorname{coth} x-\frac{1}{x}\right]
$$

où $\mathfrak{H}_{\mathrm{s}}=N \mu$ représente le "moment de saturation" mesuré à très basse température et où

$$
x=\frac{\mu B}{k T}=\frac{\mathcal{M}_{\mathrm{s}}}{R} \frac{B}{T} .
$$

La fonction $\mathfrak{f}(x)=\operatorname{coth} x-\frac{1}{x}$ est appelée « fonction de Langevin ». Elle se réduit, à des températures suffisamment élevées $[\mu B / k T=x \ll 1]$, à l'expression simple $x / 3$ et la relation devient alors : $\mathcal{M}=\chi_{0} B$, la susceptibilité $\chi_{0}$ étant égale à $\frac{1}{3} M_{\mathrm{s}}^{2} / R T$. Langevin retrouve ainsi la loi de Curie, mais montre quà très basse température un phénomène de saturation doit se produire.

Lorsque plus tard les températures de l'hélium liquide devinrent accessibles, cette prévision se confirma, mais le moment de saturation qu'on trouva, dans la plupart des cas étudiés, était différent de celui prédit par la théorie de Langevin. Le désaccord devait être expliqué par la théorie des quanta. Il était réservé à Léon Brillouin de montrer que l'expression de la susceptibilité est à modifier sous la forme suivante :

$$
\chi_{0}=\frac{1}{3} \frac{J+1}{J} \frac{M_{s}^{2}}{R T}
$$

$J$ s'appelle le nombre quantique interne de l'ion paramagnétique.

Conformément au principe de correspondance, on retrouve l'expression classique de Langevin lorsqu'on fait tendre $J$ vers linfini.

5. De nouvelles techniques confirment la réalité moléculaire. - Le nombre d'Avogadro ayant été ainsi déterminé par de nombreuses méthodes, avec une bonne précision, de nouvelles techniques devaient être mises au point, confirmer la réalité moléculaire et connaitre au $\mathrm{xx}^{\mathrm{e}}$ siècle un développement intéressant. Nous allons mentionner quelques-unes de ces techniques.

La première d'entre elles est l'étude des lames minces formées par des molécules à longue chaîne hydrocarbonée, telles que les lames de bulles de savon dont les couleurs d'interférence permettent d'évaluer l'épaisseur. L'étude de ces lames à la surface d'un 
liquide montra que les molécules d'acide gras, comme par exemple l'acide palmitique $\mathrm{CH}_{3}\left(\mathrm{CH}_{2}\right)_{14} \mathrm{COOH}$ sont susceptibles de s'étaler sur l'eau en lames d'une seule couche de molécules jointives dressées normalement à la surface et ancrées dans l'eau par leur extrémité acide hydrophile. En France, le botaniste Henri Devaux mit au point des méthodes d'une élégante simplicité pour mesurer l'épaisseur de ces lames et étudier leurs propriétés. Aux Etats-Unis, Irving Langmuir développa d'intéressantes techniques pour aborder leur étude thermodynamique.

Une autre technique qui tira parti de la réalité moléculaire est l'étude des gaz sous très faible densité, lorsque le libre parcours moyen devient de l'ordre de grandeur des dimensions du récipient. Ce régime de gaz raréfié appelé « régime moléculaire» se distingue du régime gazeux normal par un certain nombre de propriétés spécifiques dont l'étude a été approfondie par le physicien danois Martin Knudsen. Ce dernier a rendu compte de ses recherches dans une belle conférence présentée au cinquantenaire de la Société Française de Physique à laquelle nous renvoyons le lecteur.

Enfin une dernière technique, liée d'ailleurs à la précédente, a été la production des jets atomiques et moléculaires dans le vide, inaugurée par Louis Dunoyer à Paris en 1911. La figure 3 montre comment il obtint

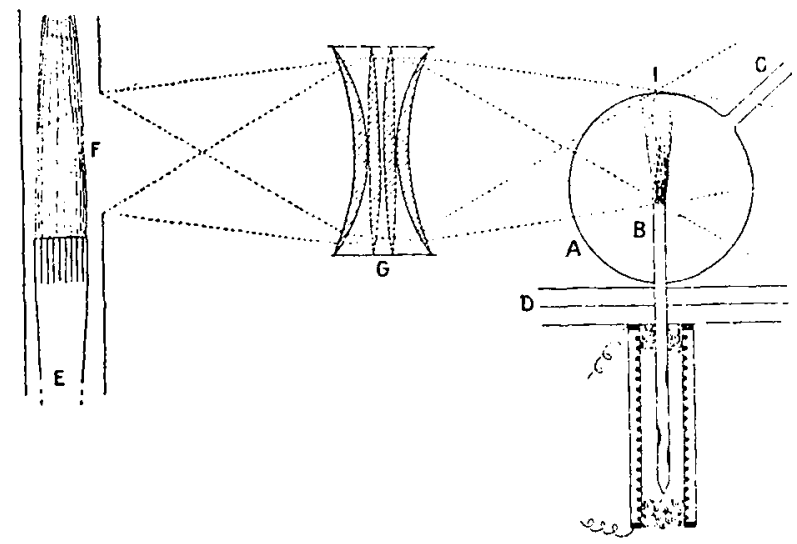

FIG. 3. - Formation et visualisation d'un jet d'atomes de sodium (d'après L. Dunoyer). Le jet est éclairé par la lumière d'une flamme de sodium qui excite la résonance optique des atomes.

un pinceau d'atomes de sodium dans le vide, ces atomes se déplaçant sur des trajectoires rectilignes grâce à leur énergie de translation d'origine thermique. Les atomes du jet peuvent être rendus visibles grâce au phénomène de résonance optique découvert par Wood.

Un moyen simple pour réaliser un pinceau atomique dans le vide consiste à utiliser un tube relié à la pompe à vide et divisé en trois compartiments successifs communiquant par deux trous $S_{1}$ et $S_{2}$ (Fig. 4). Le compartiment $\mathrm{A}$ contient un dépôt métallique et est chauffé de façon à y réaliser une pression de vapeur saturante de l'ordre de $10^{-4}$ à $10^{-3}$ torr. Les atomes s'échappant du trou $S_{1}$ pénètrent dans le comparti-

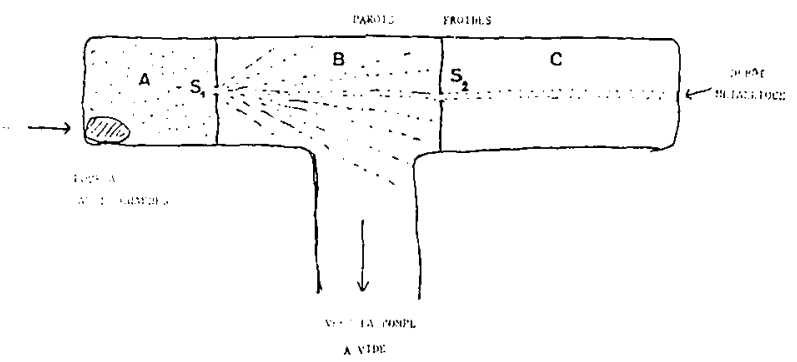

FIG. 4. - Schéma de coupe d'un appareil à jet en verre.

ment $\mathrm{B}$ à parois froides, s'y propagent dans toutes les directions et se déposent au point d'impact sur la paroi. Les quelques rares atomes dont les trajectoires sont alignées dans la direction $S_{1}, S_{2}$ vont pénétrer par le trou $\mathrm{S}_{2}$ dans le compartiment $\mathrm{C}$ et y former un pinceau d'atomes de faible ouverture angulaire. Sur la paroi de verre opposée aux trous, on observe alors un dépôt dont les dimensions renseignent sur la largeur du pinceau déterminé par la finesse des trous. On peut remplacer les deux trous par deux fentes rectilignes parallèles $F_{1}$ et $F_{2}$ afin d'obtenir sur la cible un dépôt filiforme.

On peut regretter que la technique inventée par Dunoyer n'ait pas été davantage connue en France. Le développement de cette technique à l'étranger a conduit à des résultats d'un très grand intérêt pour la physique atomique.

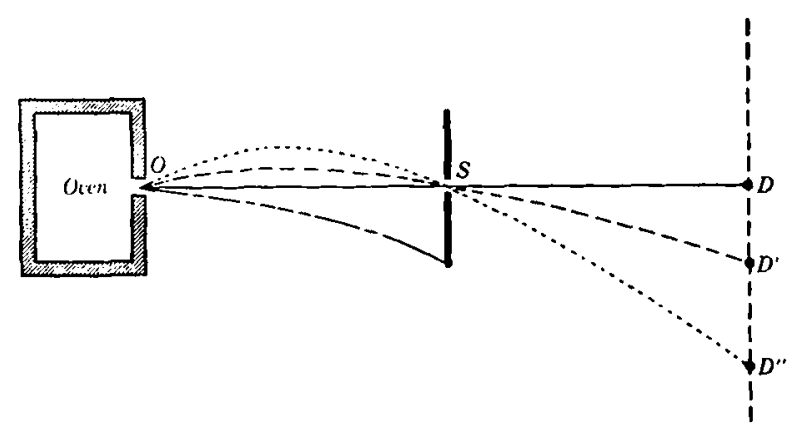

Fig. 5. - Jet horizontal d'atomes de césium dont les trajectoires sont incurvées par la pesanteur (d'après Stern, Simpson et Estermann).

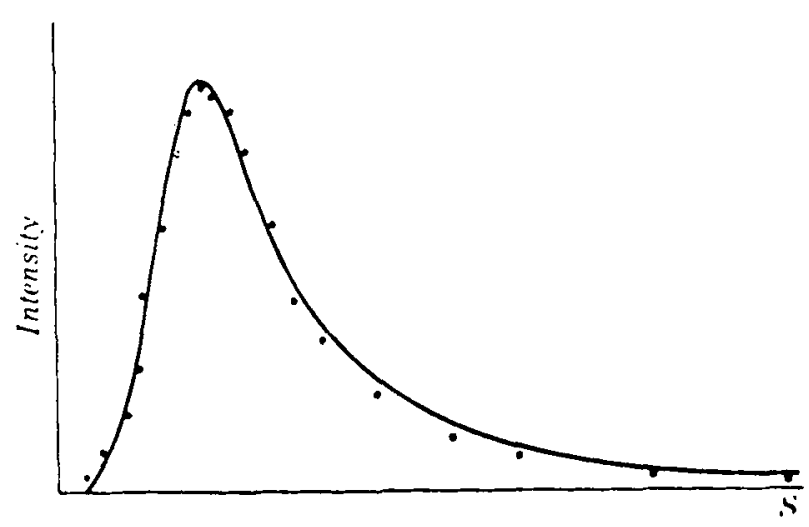

Fig. 6. - Confrontation des résultats expérimentaux avec la courbe représentant la loi de distribution des vitesses de Maxwell. 
En 1920 Otto Stern, en Allemagne, a utilisé la technique des jets pour mesurer la répartition des vitesses de translation des atomes du jet. Il a pu ainsi vérifier expérimentalement la loi de distribution de Maxwell. Il a dans la suite perfectionné cette mesure avec ses collaborateurs. Dans une de ces expériences, il a tiré parti de l'étalement vertical, sous l'effet de la pesanteur, d'un pinceau horizontal d'atomes de césium (Fig. 5). La figure 6 présente la confrontation entre la courbe théorique de Maxwell et les faits expérimentaux. Nous reviendrons plus loin sur d'autres applications de la technique des jets.

6. Les propriétés des atomes. - La réalité des atomes ayant été bien établie, les physiciens du $\mathrm{xx}^{\mathrm{e}}$ siècle se sont attachés à préciser les propriétés externes et internes de ces atomes, à connaitre leur constitution, leur structure.

Une série de remarquables découvertes faites vers la fin $\mathrm{du} \mathrm{XIX}^{\mathrm{e}}$ siècle devait inaugurer ce développement : découverte de l'électron et reconnaissance de sa présence à l'intérieur des atomes, découverte des rayons $\mathrm{X}$ par Roentgen, découverte de la décomposition magnétique des raies spectrales par Zeeman, découverte de la radioactivité par Henri Becquerel et Pierre et Marie Curie. Le développement de la physique nucléaire devait conduire Rutherford, en 1910, à partir de l'étude de la diffusion des rayons $\alpha$ par la matière, à avancer sa théorie de l'atome nucléaire : la masse de l'atome étant concentrée dans un noyau positif de très petites dimensions au centre de l'atome et ce noyau étant entouré d'électrons négatifs. L'étude de la structure des noyaux atomiques est hors du sujet de cet exposé. Même en ce qui concerne les propriétés du "cortège électronique», nous ne pouvons que résumer brièvement les étapes essentielles des progrès de nos connaissances.

Les données les plus nombreuses sur les atomes et les molécules ont été apportées par la spectroscopie dont le domaine d'investigation s'est étendu à partir du spectre visible du côté des fréquences croissantes vers l'ultraviolet proche et lointain et vers les rayons $\mathrm{X}$, du côté des fréquences décroissantes vers l'infrarouge proche et lointain et vers les radio-fréquences. La théorie des quanta des atomes, introduite par Niels Bohr, en 1913, a permis d'interpréter l'énorme matériel de données accumulé par la spectroscopie atomique. Dans cette théorie, les atomes sont caractérisés par une suite de niveaux d'énergie discontinus, et les raies spectrales correspondent à des transitions entre deux de ces niveaux. Chacun de ces niveaux est caractérisé non seulement par une certaine valeur $E_{J}$ de son énergie, mais par un moment cinétique $P_{J}$ auquel est associé un moment magnétique $\mu_{J}$. Le moment cinétique est un multiple entier ou demi-entier d'une unité quantique de moment cinétique égale à $h=h / 2 \pi$ ( $h=$ quantum d'action de Planck), et se met sous la forme $P_{J}=J \hbar$, le nombre $J$, entier ou demi-entier, étant appelé nombre quantique interne. Le moment magnétique $\mu_{J}$ qui lui est associé s'exprime par la relation :

$$
\mu_{J}=g J \mu_{\mathrm{B}}
$$

où $\mu_{\mathrm{B}}=\frac{|e|}{2 m_{0}} \hbar$ est l'unité atomique du moment magnétique ou «magnéton de Bohr » et $g$ un nombre fractionnaire simple appelé "facteur de Landé », - $\left(e / m_{0}\right)$ est le rapport de la charge à la masse de l'électron. Le moment magnétique $\boldsymbol{\mu}_{J}$ de l'atome est un vecteur axial porté par le même axe que le vecteur moment cinétique $\mathbf{P}_{J}$. Nous adoptons la convention du signe $g>0$ lorsque $\boldsymbol{\mu}_{J}$ et $\mathbf{P}_{J}$ sont des vecteurs de sens opposés.

On appelle rapport gyromagnétique le rapport

$$
\gamma=-\frac{\boldsymbol{\mu}_{J}}{\mathbf{P}_{J}}=g \frac{|e|}{2 m_{0}} .
$$

Lorsqu'on applique à l'atome un champ magnétique $H_{0}\left({ }^{2}\right)$ un niveau d'énergie $E_{J}$ se subdivise en $2 J+1$ niveaux équidistants d'énergies

$$
E_{J, m}^{\prime}=E_{J}+m g \mu_{\mathrm{B}} H_{0}
$$

le nombre $m$ appelé nombre quantique magnétique pouvant prendre toutes les valeurs différant d'une unité entre $-J$ et $+J$.

Chacun des sous-niveaux caractérisé par une valeur du nombre $m$ s'appelle sous-niveau magnétique ou sous-niveau Zeeman.

L'angle 0 entre le vecteur champ $\mathbf{H}$ et le vecteur $\mathbf{P}_{J}$ est donné par $\cos \theta=m / J$ et la projection du vecteur $\mu_{j}$ sur la direction du champ a pour valeur

$$
\mu_{n}=-\mu_{J} \cos \theta=-m g N_{\mathrm{B}} .
$$

L'angle $\theta$ ne peut prendre qu'une suite discontinue de $2 J+1$ valeurs, et ce fait constitue la quantification spatiale des moments atomiques. Un état atomique pour lequel $J=0$ (ce qui entraîne $P_{J}=0$ et $\mu_{J}=0$ ) est appelé diamagnétique, un état $J \neq 0$, pour lequel $P_{J} \neq 0$ et $\mu_{J} \neq 0$ est dit paramagnétique.

La décomposition magnétique des raies spectrales (effet Zeeman) est une conséquence de la décomposition magnétique des niveaux d'énergie. Comme les valeurs du facteur $g$ pour les deux niveaux d'une transition spectrale sont, en général, différentes, la décomposition spectrale des raies est un phénomène complexe, et l'on parle d'effets Zeeman anormaux.

L'étude de ces effets Zeeman a suggéré, en 1925, à Uhlenbeck et Goudsmit l'idée que l'électron possède un moment cinétique propre égal à $\frac{1}{2} \hbar$ auquel est associé un moment magnétique égal au magnéton de Bohr $\mu_{B}$. Cette double propriété s'appelle le "spin de l'électron " auquel correspond un rapport gyromagnétique $|\ell| / m_{0}$ et un facteur de Landé $g_{s}=2$.

Vers la même époque, Pauli formula son célèbre principe d'exclusion. "L'état d'un électron dans un atome est caractérisé par quatre nombres quantiques:

(2) En réalité la notation habituelle $\mathbf{H}_{0}$ s'applique au vecteur « induction magnétique ». 
le nombre entier $n$ appelé quantum principal et caractérisant la couche d'énergie à laquelle appartient l'électron, le nombre entier l, ayant pour valeurs extrêmes 0 et $(n-1)$, soit $n$ valeurs possibles qui déterminent le moment cinétique $l \hbar$ attaché à la trajectoire électronique, le nombre entier $m_{l}$ qui peut prendre $(2 l+1)$ valeurs entières de $-l \grave{a}+l$ et qui correspond à la projection du vecteur $l$ sur un axe de quantification spatiale, enfin le nombre $m_{\mathrm{s}}$ qui peut prendre l'une des deux valeurs $+\frac{1}{2}$ ou $-\frac{1}{2}$ et qui correspond à la projection du vecteur spin de l'électron $s=\frac{1}{2} \hbar$ sur ce même axe de quantification ). Selon le principe d'exclusion deux électrons du cortège ne peuvent pas avoir les mêmes quatre nombres en commun. Le principe d'exclusion détermine l'arrangement des électrons d'un atome en «couches» successives caractérisées chacune par le nombre n ( $n=1,2,3, \ldots$ etc.) et cette disposition entraine la formation du tableau périodique des éléments.

L'étude des raies spectrales avec des spectroscopes de haute résolution, et en particulier avec l'interféromètre Fabry-Perot, a révélé une structure de ces raies appelée "structure hyperfine ». Pauli a attribué, en 1925, cette structure à l'existence d'un spin des noyaux atomiques, qui lui aussi correspond à l'association d'un moment cinétique $I / h$ (où $I$ est le nombre de spin nucléaire entier ou demi-entier) à un moment magnétique nucléaire. Celui-ci est d'un ordre de grandeur mille fois plus petit que le magnéton de Bohr et s'exprime en une unité

$$
\mu_{N}=\mu_{\mathrm{B}} \frac{m_{0}}{M_{I I}}
$$

où $m_{0} / M_{\|}$est le rapport de la masse de l'électron à la masse du noyau d'hydrogène ou proton.

Les nombres de spins nucléaires $I$ et les moments magnétiques nucléaires $\mu_{I}$ sont connus pour la plupart des noyaux atomiques à l'heure actuelle, et cette connaissance est précieuse pour la compréhension de la structure des noyaux. C'est ainsi que le proton ou noyau d'hydrogène possède les valeurs $I=\frac{1}{2}$ et $\mu_{I}=2,79278 \mu_{N}$ alors que les valeurs correspondantes pour le neutron sont $I=\frac{1}{2}$ et $\mu_{I}=-1,928 \mu_{N}$.

7. Confirmations expérimentales. - La théorie de Bohr qui s'est développée essentiellement grâce aux

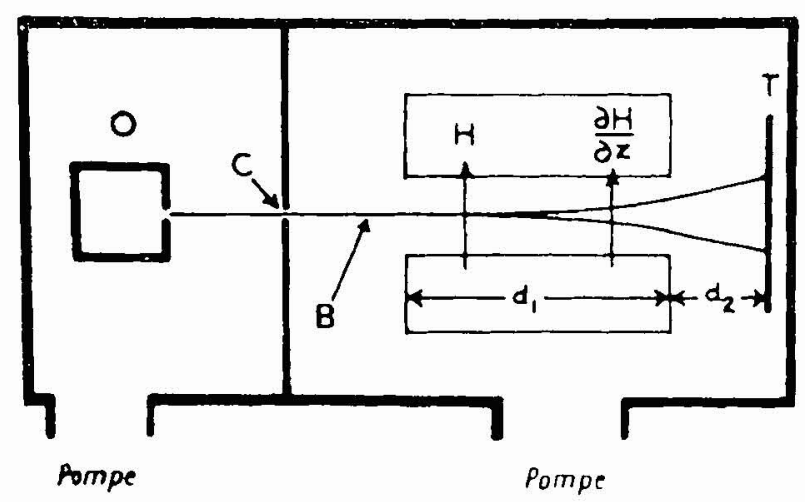

FIG. 7. - Schéma de l'expérience de Stern et Gerlach.

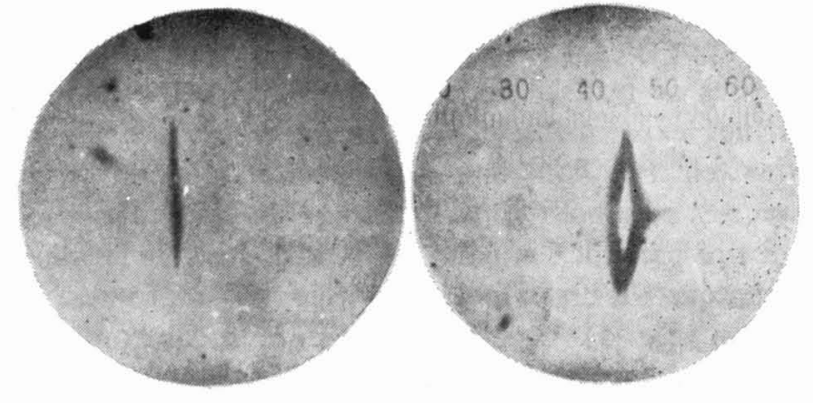

FIG. 8. - Trace d'un jet d'atomes d'argent (d'après Stern et Gerlach) : a) en absence du champ, b) en présence du champ qui qui dévie les atomes.

données de la spectroscopie, a été confirmée par les expériences de Franck et Hertz qui, en bombardant, à partir de 1913, des atomes avec des électrons monocinétiques, ont mis en évidence les niveaux d'énergie discontinus des atomes et ont déterminé leurs potentiels d'excitation à partir du niveau fondamental. En 1922, l'expérience de Stern et Gerlach, une des plus belles applications de la technique de Dunoyer à la physique atomique, confirma l'existence de la quantification spatiale et conduisit à des mesures des moments magnétiques des atomes et des noyaux atomiques. Dans cette expérience un jet d'atomes paramagnétiques (atomes d'argent ou atomes alcalins) traversa l'entrefer d'un aimant où règne un fort gradient de champ $\delta H / \delta Z$ dans une direction $Z$ qui est celle du champ et qui est normale à l'axe du jet. Si nous désignons comme précédemment la projection du moment magnétique $\mu_{J}$ de l'atome dans la direction $Z \mathrm{du}$ champ par $\mu_{\| i}$, la force qui s'exerce sur un atome du jet est donnée par

$$
F_{Z}=\mu_{H} \frac{\delta H}{\delta Z}=-m g \mu_{\mathrm{B}} \frac{\delta H}{\delta Z} .
$$

Les atomes qui sont dans des états de quantification spatiale $m$ différents subissent des forces différentes, donc éprouvent des déviations différentes, et le jet se décompose ainsi en $(2 J+1)$ composantes différentes. Avec des atomes d'argent et avec des atomes alcalins les auteurs ont observé un dédoublement du jet. Ils ont conclu que pour les états fondamentaux de ces atomes, le nombre $J=\frac{1}{2}$. L'évaluation quantitative de la déviation a montré que $\mu_{J}=\mu_{\mathrm{B}}$.

8. Applications de la spectroscopie des radiofréquences. - Cette expérience de Stern et Gerlach a connu de nombreux affinements par Stern et ses collaborateurs. En 1937, le professeur Rabi à l'Université Columbia de New York, qui s’était initié à la technique des jets à Hambourg à l'Institut de Stern, a appliqué aux atomes et molécules paramagnétiques d'un jet, sur la suggestion de Gorter, un champ alternatif de radiofréquence en présence d'un champ magnétique constant et homogène. Il a observé ainsi les premières transitions de résonance magnétique grâce à la modification des trajectoires des atomes 


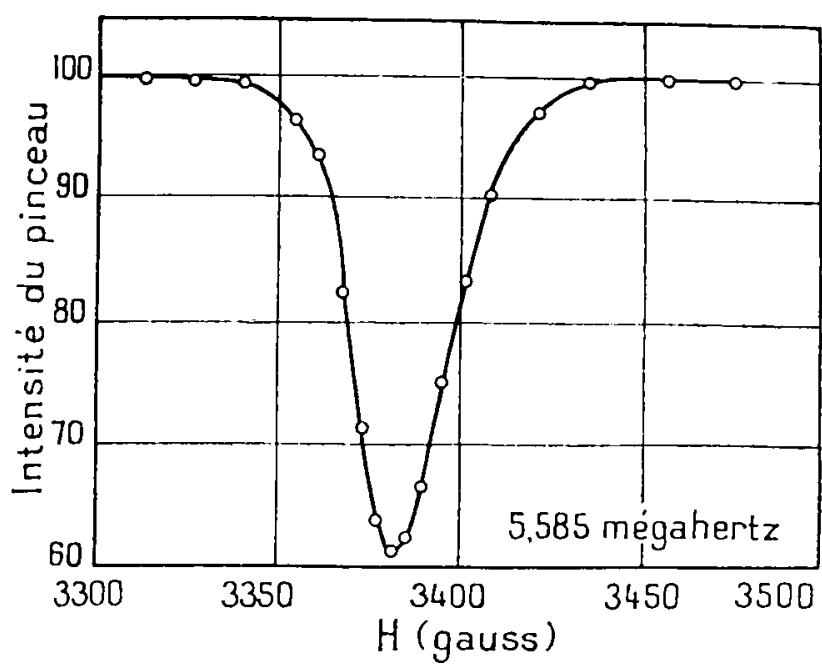

FIG. 9. - Une des premières courbes de résonance magnétique nucléaire obtenue par Rabi, celle de $7 \mathrm{Li}$ dans $\mathrm{LiCl}$.

ayant subi une telle transition. A partir de cette date, la méthode de spectroscopie de radiofréquence appliquée à des jets atomiques et moléculaires a connu de nombreux développements. Elle a permis de mesurer des intervalles d'énergie et des moments magnétiques avec une précision remarquable et de découvrir des anomalies d'un grand intérêt théorique.

Nous allons nous borner à citer quelques exemples de résultats obtenus. L'intervalle de structure hyperfine du césium 133 a été mesuré dans l'état fondamental de cet atome avec une précision de 10 chiffres significatifs. Etant donné cette précision, la fréquence de cet intervalle a été adoptée comme fréquence étalon :

$$
\Delta v_{\mathrm{Cs}_{\mathrm{s}}}=9192631770,00 \mathrm{~Hz}
$$

et l'unité de temps a été définie à partir de cette fréquence, une seconde étant, par définition, égale à la durée de 9192631770 périodes de la vibration hyperfine de $\mathrm{Cs}^{133}$. L'appareil qui permet de se référer à cette fréquence étalon s'appelle une « horloge atomique $»$.

En sélectionnant dans un pinceau moléculaire les molécules dans un état d'énergie qui représente le niveau supérieur d'une transition hertzienne, et en introduisant ces molécules riches en énergie dans une cavité accordée, il a été possible d'amorcer l'oscillation électromagnétique de la cavité et de créer ainsi un auto-oscillateur quantique appelé MASER. Le premier en date de ces « masers » réalisé en 1954 pár Townes, a été le maser à ammoniac. Dans la suite, et en s'inspirant du même principe, Ramsay, au MIT, a réalisé un maser à hydrogène atomique oscillant sur la fréquence hyperfine de l'atome d'hydrogène à l'état fondamental. Cette fréquence, comparée à celle de l'horloge atomique au césium, a été trouvée égale à

$$
\Delta v_{H}=1420405751,800\left( \pm 28 \times 10^{-3}\right) \mathrm{Hz} .
$$

Enfin la fréquence hyperfine de l'atome de tritium comparée à celle d'hydrogène normal a fourni le rapport

$$
\frac{\Delta v_{T}}{\Delta v_{I I}}=1,067794514973
$$

rapport déterminé avec une précision de $5 \times 10^{-12}$ qui représente, sans doute, la précision la plus élevée atteinte jusqu'ici dans une mesure physique.

En utilisant un jet d'hydrogène atomique contenant des atomes à l'état métastable $2{ }^{2} \mathrm{~S}_{1 / 2}$ Lamb et Retherford ont réussi à mesurer, en 1947, par des techniques de résonance hertzienne, l'écart entre les niveaux de structure fine $2{ }^{2} \mathrm{~S}_{1 / 2}$ et $2{ }^{2} \mathrm{P}_{1 / 2}$ de l'atome d'hydrogène. Ils ont trouvé pour valeur de cet écart

$$
\Delta v_{L}=1058,32 \mathrm{MHz} .
$$

Or, d'après la théorie quantique de Dirac, ces deux niveaux d'énergie auraient dû coïncider. L'existence du «Lamb-shift » a donc nécessité un affinement de la théorie. A l'heure actuelle cet écart est pleinement expliqué par la théorie de l'électrodynamique quantique développée par Feynmann et Schwinger. Cette théorie prévoit également que le facteur de Landé du spin de l'électron n'est pas exactement égal à 2 mais égal à $2(1+\varepsilon)$ où $\delta$ est un terme correctif de l'ordre de $10^{-3}$. Cette anomalie a été mise expérimentalement en évidence par Kusch et Foley grâce à une technique de jet atomique. Le facteur correctif $\mathcal{E}$ est aujourd'hui connu avec précision. Il est égal à

$$
\varepsilon=\frac{g-2}{2}=10^{-3} \times 1,1596577\left( \pm 35 \times 10^{-7}\right) .
$$

Cette valeur coïncide remarquablement avec la valeur théorique calculée par l'électrodynamique quantique.

9. Les théories quantiques introduisent de nouveaux concepts. - La théorie des quanta d'origine germanique a mis longtemps à s'acclimater en France. Nous devons rendre hommage à Edmond Bauer et à Eugène Bloch qui, par leur enseignement, firent connaître les idées quantiques aux jeunes scientifiques français. Le concept de la nature ondulatoire de la matière, introduit par Louis de Broglie, en 1923, fut développé par Schrödinger dans sa mécanique ondulatoire à partir de 1926, en compétition avec la mécanique quantique inaugurée par Heisenberg, Born et Jordan. Ces percées devaient donner à la théorie quantique un épanouissement qui allait culminer dans l'électrodynamique quantique déjà mentionnée. Les recherches théoriques et les recherches expérimentales se complétèrent pour saisir la réalité atomique et pour conduire à une connaissance des propriétés atomiques avec une précision insoupçonnée peu d'années auparavant et dont nous avons donné des exemples.

Mais ces nouvelles mécaniques devaient modifier profondément le concept même de "réalité atomique" et montrer que les particules à l'échelle atomique ont des propriétés très différentes des objets que nous 
percevons directement par nos sens ou des « points matériels » avec lesquels opère la mécanique rationnelle.

Cette évolution est due à un curieux retour vers les exigences $\mathrm{du}$ positivisme scientifique qui demande qu'on élimine de la science tout «mòdèle » qui n'est pas accessible à l'expérience. Les modèles macroscopiques imaginés pour représenter les atomes et es molécules avaient servi d'auxiliaires précieux aux physiciens atomistes. Les chimistes également se servent constamment de modèles moléculaires qui leur ont rendu de grands services pour édifier la stéréochimie et pour interpréter certaines propriétés physiques des molécules, comme le pouvoir rotatoire, grâce à des considérations de symétrie. Les cristallographes de leur côté ont tiré un grand parti des modèles de structure cristalline. Rien d'étonnant donc que les physiciens eux aussi aient pensé à se servir de modèles des atomes à l'aide desquels il était possible d'interpréter les propriétés internes et externes de ces atomes. Rutherford avait montré que la masse du noyau était localisée dans un très petit volume central de dimension de l'ordre de $10^{-13}$ à $10^{-12} \mathrm{~cm}$ alors que le diamètre même des atomes était de l'ordre de $10^{-8} \mathrm{~cm}$. Enfin la théorie de Bohr considérait l'atome comme un système planétaire dans lequel les électrons gravitent autour du noyau sur des «trajectoires » à caractère périodique, comme des planètes gravitent autour du soleil.

Mais le fait que, conformément aux prévisions de la mécanique ondulatoire, un pinceau d'électrons puisse, dans l'expérience de deux fentes de Young donner

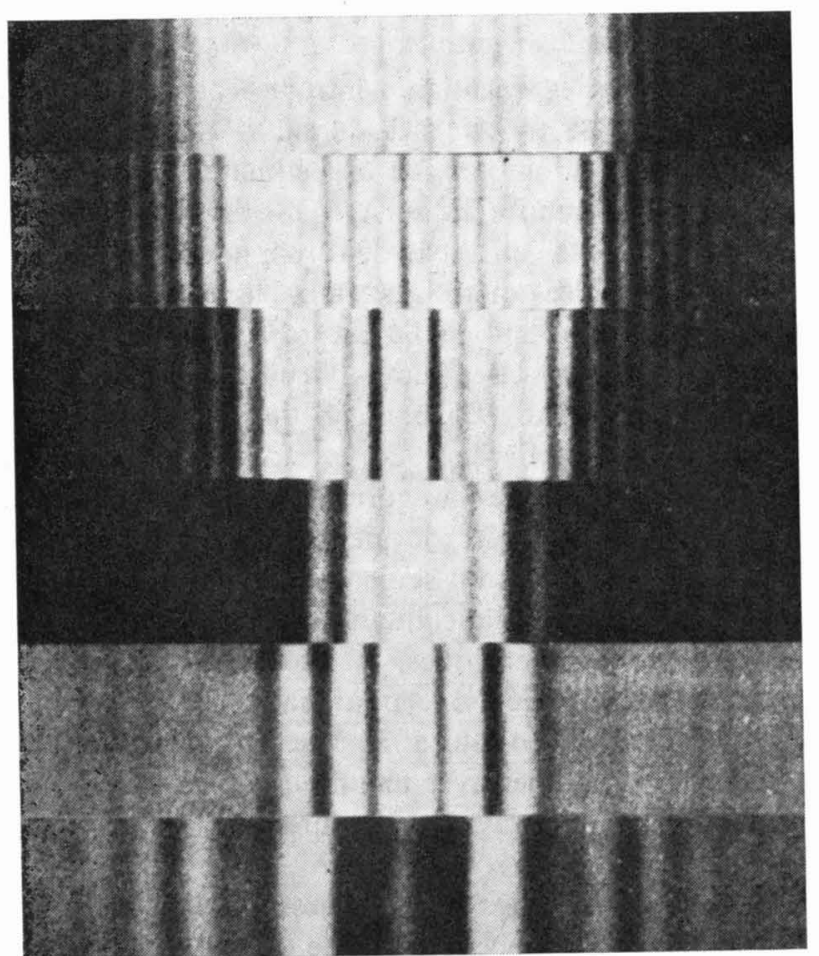

Fig. 10. - Franges dinterférence produites par des électrons dans l'expérience de deux fentes de Young (d'après Faget). lieu à la production de franges d'interférence - exactement comme le fait un pinceau de lumière - montre que l'évolution spatio-temporelle d'un électron ne se fait pas sur une «trajectoire » où à chaque instant la position spatiale est connue en fonction du temps. Heisenberg fit remarquer qu'une " trajectoire électronique » à l'intérieur de l'atome n'avait pas de réalité physique et échappait à toute observation. Si la notion de «permanence spatio-temporelle » d'une particule était ainsi mise en question, la notion d' « individualité » ou « discernabilité » qui est une propriété des objets macroscopiques, devait à son tour être abandonnée pour les particules à l'échelle atomique. Cette nécessité résulte du comportement statistique des molécules, et en particulier du simple fait que la relation de Boltzmann-Planck $S=k$ in $W$ ne conduit à une entropie qui possède la propriété de grandeur extensive que si l'on fait cette concession.

Ces considérations devaient se concrétiser dans le principe d'indétermination de Heisenberg (1927) aboutissement d'une analyse critique du processus de mesure des coordonnées spatio-temporelles d'une particule. Ce principe s'exprime par les relations bien connues :

$$
\begin{aligned}
& \Delta x \cdot \Delta p_{x} \approx \hbar \\
& \Delta E \cdot \Delta t \approx \hbar .
\end{aligned}
$$

Appliquons ce principe à l'atome d'hydrogène dans son état fondamental, formé par l'association de deux particules, un proton chargé positivement et un électron chargé négativement. Si ces deux particules chargées se comportaient comme des points matériels de la mécanique rationnelle, un tel système devrait posséder à chaque instant un moment électrique dipolaire, et ce moment devrait évoluer périodiquement en fonction du temps. Or, toutes les observations montrent que cet atome, dans son état fondamental possède la symétrie sphérique (isotropie) caractéristique d'un état $S$.

Sachant que l'électron est à l'intérieur de l'atome, nous connaissons son abscisse avec une précision de l'ordre de l'angström. Donc $\Delta x \leqslant 10^{-8} \mathrm{~cm}$. Il en résulte que nous devons avoir

$$
\Delta p \geqslant \frac{10^{-27}}{10^{-8}}=10^{-19} \text { C. G.S . }
$$

Mais

$$
\Delta p=m_{0} \Delta v \quad \text { et } \quad m_{0} \sim 10^{-27} \mathrm{~g}
$$

done

$$
\Delta v=\frac{\Delta p}{m} \geqslant 10^{8} \mathrm{~cm} / \mathrm{s} .
$$

D'après la théorie de Bohr, la vitesse même de l'électron sur sa trajectoire fondamentale est, $\alpha$ étant la constante de structure fine,

$$
v=\alpha c=\frac{3 \times 10^{10}}{137} \approx 2,2 \times 10^{8} \mathrm{~cm} / \mathrm{s} .
$$

Nous voyons donc que l'incertitude sur la vitesse 
est du même ordre de grandeur que le mođule même de cette vitesse. C'est dire que nous ne pouvons connaître, à un instant donné, ni la grandeur, ni la direction de cette vitesse, et que la «phase » du mouvement périodique est pour nous indéterminable.

Nous comprenons ainsi pourquoi l'électron de l'atome $\mathrm{H}$ ne peut pas être représenté par un point matériel évoluant sur une trajectoire classique. Il est représenté en mécanique quantique par une "fonction d'onde " $\psi(x, y, z)$ dont les propriétés spatiales, pour un état $S$, sont isotropes et dont le carré représente la "probabilité de présence » de l'électron en une petite région de l'espace définie par sa distance au noyau. Si nous voulons nous faire un "modèle " de cet atome, celui d'un "nuage de charge » entourant le noyau est le plus adéquat.

Un autre cas - plus surprenant encore - où la différence par rapport au modèle macroscopique devient patente - est celui de la molécule diatomique d'hydrogène dans son état de parahydrogène $(I=0)$ dépourvu d'énergie de rotation $(J=0)$. La fonction d'onde de cet état a des caractères d'isotropie, et cette isotropie de la molécule se manifeste dans la manière dont elle diffuse la lumière; sa raie Rayleigh de diffusion est parfaitement polarisée comme l'est celle d'un atome isotrope.

Je voudrais évoquer une dernière conséquence du principe d'indétermination : les physiciens du XIX ${ }^{e}$ siècle s'étaient abondamment servis du concept d'éther, milieu physique servant de support aux ondes lumineuses; ainsi Fresnel avait rendu compte de la diffraction en faisant intervenir des « sources secondaires » tapissant la surface de l'ouverture diffractante. L'avènement de la théorie de la relativité restreinte avait sonné le glas de l'éther, car l'existence d'un milieu physique qui devait nécessairement être au repos dans un système particulier de référence galiléen, et par conséquent en mouvement par rapport à tous les autres systèmes, semblait incompatible avec le postulat d'équivalence de tous les systèmes galiléens. Ce raisonnement était probant tant qu'on assimilait les particules d'éther à des particules obéissant aux lois de la mécanique rationnelle. Mais si l'on fait intervenir le principe d'indétermination et si l'on admet que la masse des particules d'éther - comme celle des neutrinos - est évanescente, l'indétermination de leur vitesse devient extrême et l'existence physique de telles particules devient parfaitement compatible avec l'équivalence des systèmes galiléens.

Nous voyons donc que si le physicien d'aujourd'hui ne doute plus de l'existence des atomes, il attribue aux particules à l'échelle atomique des propriétés bien différentes de celles des objets macroscopiques accessibles à nos sens. Les particules à l'échelle atomique ne possèdent pas les propriétés d'individualité et de permanence (au sens d'une trajectoire définie dans l'espace-temps) qui caractérisent les objets macroscopiques. Par contre la mécanique ondulatoire attribue à ces particules une propriété nouvelle: ce sont des entités physiques douées d'une amplitude et d'une phase. Lorsque, dans une collection de particules identiques, les phases de ces particules sont distribuées au hasard, l'ensemble des objets obéit aux lois des statistiques quantiques: l'incohérence des phases est une hypothèse fondamentale à la base de ces statistiques. Lorsque, au contraire, des relations définies existent entre les phases des particules appartenant à une collection, des propriétés nouvelles liées à la cohérence apparaissent et peuvent se manifester à l'échelle macroscopique. Un exemple aujourd'hui bien connu de ce type est la précession cohérente des composantes transversales des moments magnétiques d'une collection d'atomes autour du vecteur champ magnétique. Un autre exemple de cohérence macroscopique est le phénomène de supraconductibilité. A cette cohérence se trouve liée - comme Von Laue l'a montré pour le cas de la lumière - - une néguentropie définie. Et l'on peut se demander si le maintien de néguentropie, qui est une caractéristique essentielle des êtres vivants, n'est pas lié à une certaine cohérence des particules constitutives de ces êtres.

Mais laissons là ces spéculations et concluons : lorsqu'il y a cent ans, Max Planck qui avait alors 17 ans, hésitait sur le choix d'une carrière, l'une des sommités de la physique de ce temps à laquelle il demandait conseil, lui dit que la physique était une science achevée et qu'il n'y avait plus beaucoup d'espoir d'y faire æuvre de pionnier. Si Planck avait suivi ce conseil, le cours de la physique pendant ces cent dernières années aurait sans doute été différent. Il nous reste à souhaiter que les cent ans qui sont devant nous, nous apportent autant de surprises que le siècle qui vient de s'achever. 